\title{
The Role of Biofluid Mechanics in the Assessment of Clinical and Pathological Observations
}

\author{
Sixth International Bio-Fluid Mechanics Symposium and Workshop, March 28-30, \\ 2008 Pasadena, California
}

\begin{abstract}
Maria Siebes $^{1}$ and Yiannis Ventikos ${ }^{2}$
${ }^{1}$ Department of Biomedical Engineering and Physics, Academic Medical Center, University of Amsterdam, Meibergdreef 9, 1105 AZ Amsterdam, The Netherlands; and ${ }^{2}$ Fluidics and Biocomplexity Group, Department of Engineering Science \& Institute of Biomedical Engineering, University of Oxford, Oxford, UK
\end{abstract}

(Received 27 October 2009; accepted 2 January 2010; published online 20 January 2010)

Associate Editor Larry V. McIntire oversaw the review of this article.

\begin{abstract}
Biofluid mechanics is increasingly applied in support of diagnosis and decision-making for treatment of clinical pathologies. Exploring the relationship between blood flow phenomena and pathophysiological observations is enhanced by continuing advances in the imaging modalities, measurement techniques, and capabilities of computational models. When combined with underlying physiological models, a powerful set of tools becomes available to address unmet clinical needs, predominantly in the direction of enhanced diagnosis, as well as assessment and prediction of treatment outcomes. This position paper presents an overview of current approaches and future developments along this theme that were discussed at the 5th International Biofluid Symposium and Workshop held at the California Institute of Technology in 2008. The introduction of novel mechanical biomarkers in device design and optimization, and applications in the characterization of more specific and focal conditions such as aneurysms, are at the center of attention. Further advances in integrative modeling, incorporating multiscale and multiphysics techniques are also discussed.
\end{abstract}

Keywords-Cardiovascular fluid mechanics, Hemodynamics, Imaging, Computational modeling, Physiological modeling.

\section{ABBREVIATIONS}

FSI Fluid-structure interaction

3D Three-dimensional

Address correspondence to Maria Siebes, Department of Biomedical Engineering and Physics, Academic Medical Center, University of Amsterdam, Meibergdreef 9, 1105 AZ Amsterdam, The Netherlands. Electronic mail: m.siebes@amc.uva.nl

\begin{abstract}
PC-MRI Phase-contrast magnetic resonance imaging

HFI Helical flow index

WIA Wave intensity analysis
\end{abstract}

\section{INTRODUCTION}

Transport phenomena in general and biological fluid mechanics in particular play an important role in better understanding of clinical and pathological observations and in developing new methods for diagnosis in connection with physiological and/or computational models. Although biofluid concepts apply to numerous systems in the human body, including the ocular, ${ }^{28}$ lymphatic, ${ }^{48}$ synovial, ${ }^{79}$ cerebrospinal, ${ }^{43}$ cochlear, ${ }^{58}$ pulmonary, ${ }^{5}$ nasal, ${ }^{24,80}$ and others, ${ }^{64}$ the focus in this exposition is on the cardiovascular system. Because of its physiological predominance and clinical importance, arterial cardiovascular fluid dynamics has become the mainstay of transport phenomena biomechanics.

The topic of this position paper deals with correlation of clinical observations with the biomechanics of blood flow; in other words, we explore the link between observations of hemodynamic phenomena and mechanisms that have a strong mechanical component in their causal chain with disease manifestations or pathophysiological indicators. Apart from the fundamental interest in such correlations, there is strong practical benefit, since investigation of directly accessible or computed biofluidic observables cannot only 
lead to improved diagnostic capabilities but also to assessment and even prediction of treatment outcomes. ${ }^{47}$ Progress in imaging and measurement technology (in vivo or in silico) has dramatically increased our access to such observables in recent years.

We can exemplify the topic of this theme using an early case that is considered a classic nowadays: a correlation was proposed almost 40 years ago between atherosclerosis, considered the most prevalent vascular disease, and patterns of abnormal wall shear stress. ${ }^{12}$ This study, along with a multitude of subsequent works based on this premise, apart from shedding light on a complicated and multifactorial condition, also implies that if we can identify regions of abnormal wall shear stress (a purely mechanical measure) then we can predict where atherosclerosis may emerge (other factors contributing to the disease notwithstanding). Although this example relates specifically to the interaction of mechanical forces with the endothelium, which has evolved into a highly active research area in its own right, ${ }^{18,23}$ it serves to clarify the central theme of this paper: by establishing links of biofluid observables (pressure, flow, wall shear stress, etc.) to clinical pathological or functional observables (vascular disease, organ perfusion, cardiac performance, etc.), we utilize the former as a predictor-precursor of the latter. Especially for early stages of disease, it is hoped that such mechanical biomarkers may serve as early risk assessment for conditions that have some biofluidic component. ${ }^{31}$ Observations of this nature have triggered a host of similar assertions, and efforts to build more integrative and comprehensive models evolving around such biofluid foundations are now taking place. These links also provide important and much needed tools to support clinical decision-making in diagnosis, assessment of therapeutic interventions, and optimization of cardiovascular devices. ${ }^{61}$

To summarize, this theme explores the congruence of cardiovascular pathophysiology and the techniques and know-how to measure, or to compute, related biophysical parameters. By bringing those two tracks closer together, we envisage developing better diagnostic and prognostic tools for the cardiovascular system, where the hemodynamic component is given due importance, both as a contributing mechanism but also as a predictor of physiological behavior.

In the following sections, we shall give a brief outline of recent progress, as reported at this symposium. Any such exposition, given the vibrancy and productivity of the field, cannot be complete or even adequate, and we aim not at such coverage. We close with an outlook on future developments and recommendation of priority objectives for the integration of biofluid-physiological modeling in healthcare applications.

\section{BIOFLUID MODELING IN STUDYING CARDIOVASCULAR DEVICES}

The correlation of biofluid with clinical observations finds direct and promising application in the case of devices, especially implantable devices. As reported also elsewhere in this issue, a very substantial effort is being made to marry vasculature and implants in imaging and computational fluid dynamics to derive indicators for the efficacy, safety, and performance of heart valves, stents, coils, or grafts.

Major advances have been reported recently toward developing predictive fluid-structure interaction (FSI) algorithms that can simulate prosthetic heart valve hemodynamics at physiologic conditions and at resolution sufficiently fine to probe the links between hemodynamics and blood-cell damage. ${ }^{32,33,65}$ While heart valve studies (natural and artificial) focused mostly on the blood hemodynamics of the problem up until the recent past, an emerging trend is observed for additional mechanisms like thrombosis, hemolysis, calcifications etc., to be included in such simulations. ${ }^{25}$ This type of multiphysics-enhanced modeling is not confined to the implants field, as we shall see in the sequel. Experimental measurements of vorticity dynamics downstream of a bileaflet mechanical valve in a straight axissymmetric vessel have been reproduced in such numerical simulations with very good fidelity. $^{20}$ The simulations also provided the first glimpse into the complex, 3D hemodynamic environment experienced by blood cells especially during the decelerating flow phase when the flow transitions to turbulence and resolved a number of subtle features of experimentally observed valve kinematics, such as the asymmetric opening and closing of the leaflets and the leaflet rebound during closing. ${ }^{9}$ Results of highresolution FSI simulations of a mechanical bileaflet valve in a curved, anatomic aorta have shown the important role the aortic geometry plays on both the flow patterns and the leaflet kinematics. ${ }^{8,10}$ Computational fluid dynamics was also used to investigate the hemodynamics through a transcatheter aortic valve at different stages of sclerotic degeneration. ${ }^{26}$ The unique geometry of these stented bioprosthetic valves resulted in a large increase in peak wall shear stress on the leaflets with advanced degeneration. The orientation of the leaflets with respect to the aortic root sinus at the time of implantation may play an important role in optimizing leaflet kinematics and the durability of these percutaneous implants. Integrative modeling of heart valves has also been used to elucidate the thrombogenicity of specific devices due to shear stresstriggered platelet activation. ${ }^{25}$

It is generally accepted that in silico or in vitro optimization of devices is only the first step in the 
effort of improved clinical outcome. A parallel route is followed by studies that attempt to examine the performance of devices implanted (in reality or virtually) in anatomically accurate vascular segments. Those devices are usually stents, grafts or coils (the latter used mostly for the embolization of cerebral aneurysms). Detailed discretization of several open stents for cerebral aneurysms yielded clear trends for the preferred device, at least for the particular aneurysm tested. ${ }^{60}$ For the same pathology, an attempt to utilize a multiscale approach based on porous media considerations was reported, aiming at computing the flow reduction caused by the implantation of detachable platinum coils. ${ }^{39}$ In this case also, the results obtained confirm clinical experience and practice. Similarly, the effects of theoretical stent implantation in a coronary artery were investigated recently. ${ }^{44}$ Relatively few reports are available for devices connected to the venous system; other examples relate to hemodialysis grafts $^{75}$ or arteriovenous fistulae. ${ }^{41}$

The introduction of computational techniques that are more inherently capable in handling the complexities of blood flow and its biological and biomechanical interactions should be mentioned here. An example of this type of approach is the lattice Boltzmann method, which has been used for the modeling of thrombosis ${ }^{37}$ or for the multiscale modeling of the particulate nature of blood and related flow phenomena. ${ }^{2,3,16,19,45,62}$

Research efforts such as those described above provide good correlation of simulation results with experimental and clinical data and can therefore help in the design of better devices.

\section{BIOFLUID MODELING APPLICATIONS IN 3D CURVED VESSELS}

The onset of complex hemodynamics ${ }^{50}$ is initiated by the curved, three-dimensional form of the aortic arch and coronary vessels. In particular, the ascending, transverse, and descending aorta, joined with the structures projecting into the flowing blood stream at or above the aortic valve, and the ventricular twisting and torsion during contraction ${ }^{42}$ contribute to produce a flow field characterized by helical motion in the human aorta. ${ }^{63}$

The intrinsic capability of Phase-Contrast Magnetic Resonance Imaging (PC-MRI) to map time-accurate three-dimensional flow patterns offers the possibility of analyzing local vascular hemodynamics. ${ }^{81}$ Novel approaches apply topological tools and concepts of fluid mechanics (frequently used as post-processing techniques to computational results) to MR-derived velocimetry in the aorta. ${ }^{34,52}$ Such techniques contribute to produce a flow field characterization that allows for the quantitative ranking of transient blood flow, thereby enhancing our knowledge on the potential advantages to the primary circulation arising from asymmetries and chiralism, both in the anatomy and hemodynamics.

In brief, particle traces are calculated from temporally resolved velocity data and an integrated Lagrangian-based metric, the Helical Flow Index (HFI), is derived. ${ }^{51}$ HFI is a seven-dimensional (velocity components, velocity gradients and time) index for helical flow quantification. The strength of HFI is in the fact that it represents a method for ranking the fluid dynamical behavior into vessels: while it is possible to visually differentiate two and three-dimensional, streamlined and vertical, flow patterns during the cardiac cycle, or between physiological and pathological cases, an indicator or a metric like HFI eases the level of comparison. Initial results demonstrate that the evolving helical flow structures work in order to mitigate loss of organization in the flow field and, as a consequence, transition to turbulence. This suggests the possibility that helicity formation might be the consequence of an evolutionary optimization in naturally occurring fluid transport processes in the cardiovascular system, aiming at obtaining efficient perfusion by minimizing energy dissipation and limiting atherogenic flow instabilities. In fact, many properties of systems presenting helicity are related to the reduction of non-linear processes responsible for the transfer and redistribution of energy through various scales. ${ }^{51,73}$

We expect that the use of hemodynamic synthetic descriptors as a metric for ranking the behavior of transient hemodynamics in these regions of interest will take the utility of PC-MRI techniques to a new level. Such advanced biomechanical topological biomarkers, along with methods for data handling and flow feature visualization, allow for analyzing large three-dimensional in vivo data sets and may provide useful information in diagnosis/prognosis in clinical practice.

\section{BIOFLUID APPLICATIONS IN RISK ASSESSMENT OF CEREBRAL ANEURYSMS}

The biofluid study of cerebral aneurysms deserves special mention, because of the great interest this particular pathology raises. The reasons for this include the very focal nature of the disease, that makes it amenable to biomechanical investigation, but also the fact that cerebral aneurysms highlight the importance of patient-specific disease evaluation in the formulation of healthcare policy: aneurysms are found in $2-5 \%$ of the general population, with very high mortality 
and morbidity rates in case of rupture $(30-50 \%)$. Most of these aneurysms remain asymptomatic; however, annual incident rates of subarachnoid hemorrhage are low, 2-40 per 100,000 subjects. $^{38}$ In spite of this apparent lack of clinically significant indicators, relatively recent developments in imaging have led to new healthcare policies in terms of application of medical imaging modalities: in practice, nowadays many admissions that involve any head-related symptom include MR, CT, or other scanning. This trend has led to a dramatic increase in coincidentally detected asymptomatic cerebral aneurysms and thus to significant pressure in terms of decision-making and intervention optimization. ${ }^{78}$ In effect, there is great need for answering the question: "once an asymptomatic lesion is detected, should we intervene?", based on robust decision-making know-how. Imaging and computational modeling as a suite can help offer answers to these questions, by introducing quantitative risk evaluation criteria. ${ }^{14}$

As mentioned before, a clear trend to examine and characterize biofluid behavior based exclusively on rigorously extracted geometric features is emerging. This class of techniques offers the benefit of very rapid evaluation for a large population of cases. For the case of cerebral aneurysms, the extraction of geometric indicators, looking at connecting those with flow and pathologies, has been reported. ${ }^{49}$ Techniques of this nature are now culminating into large-scale studies that hold great promise of a robust and predictive categorization of lesions, without the need for costly computational hemodynamics simulations, for aneurysms but also for a more general assessment of cerebral vasculature pathologies. ${ }^{46}$

\section{INTEGRATION OF BIOFLUID WITH PHYSIOLOGICAL MODELS}

Although the greatest utility in establishing direct correlations between biological fluid mechanics and observed pathophysiology is the predictive power that such correlations offer, it is becoming apparent that in many cases the biomechanics of fluid flow alone, although important, cannot be used to convey a comprehensive and thus prognostic picture of a particular situation. To remedy this, combinations of biofluid models with models of pertinent mechanical, biological, or physiological mechanisms are introduced. Although fluid-structure interaction frameworks incorporating vascular wall mechanics and hemodynamics have existed for some time, ${ }^{29}$ this theme is seeing substantial cross-disciplinary enrichment through vascular biology models of wall remodeling in risk assessment for both aneurysms (abdominal $^{76}$ and cerebral ${ }^{14,15,21,77}$ ) and coronary plaque rupture. ${ }^{40,53,55,56,59}$

Useful mechanistic insight can also be gained from direct hemodynamic measurements in combination with physiological modeling. Microvascular dysfunction plays an important role in the pathogenesis of myocardial ischemia but is difficult to assess in the catheterization laboratory. Coronary blood flow is highly dependent on cardiac-coronary interaction and typical "out-of-phase" pressure and flow waveforms result from the combined action of dynamic changes in aortic pressure and the compression/relaxation exerted by the beating heart on the intramural coronary vessels that penetrate the heart muscle. ${ }^{66}$ Coronary hemodynamic waveforms are furthermore strongly influenced by the presence of atherosclerotic stenoses and the vasodilatory status of the coronary microcirculation. However, clinical assessment of coronary artery disease and microvascular function is traditionally limited to the analysis of beat-averaged hemodynamic data ${ }^{67}$ and the information contained in pulsatile characteristics of coronary flow velocity and pressure is still not well utilized. Wave intensity analysis (WIA) is a promising time-domain method that interprets incremental changes in pressure and velocity signals as the local sum of energies carried by incident forward and backward traveling waves. ${ }^{57}$ This method has great potential of developing into a powerful tool for assessment of the human coronary microcirculation, as it distinguishes between forward waves generated by the variations in aortic pressure and backward waves arising from the microcirculation due to the mechanical actions of the cardiac muscle. ${ }^{7}$ WIA has been successfully used to elucidate waveform generation in the epicardial coronary vessels of humans ${ }^{22,36}$ and was shown to provide quantitative and detailed information for diverse cardiovascular pathologies in the systemic circulation, based on noninvasive measurements in the clinical setting. ${ }^{71}$ Combining physiological modeling and clinically accessible hemodynamic measurements can play a key role not only in diagnosis and treatment evaluation, but also in the validation of image-based computational models.

\section{CAVEATS REGARDING MEDICAL IMAGE-BASED CFD MODELS}

The proliferation and availability of imaging modalities and computational techniques is bringing drastic change in clinical practice. Biomechanical indicators, when blood flow is concerned, are derived predominantly through a combination of imaging and computer modeling. Computational simulation models based on anatomically derived vascular geometries are 
increasingly being developed in the pursuit of enhanced clinical relevance nowadays. ${ }^{17,68,69}$ This has interesting implications in the sense that computational simulation is perceived more as a meta-modality, i.e., a post-processing value-adding filter to clinical imaging rather than a knowledge generator in its own right, although the latter does indeed offer great insight in complex biofluidic phenomena. ${ }^{6}$ However, in spite of the direct clinical appeal that such anatomically accurate (or more correctly, anatomically derived) patient-specific models present us with, they are not without their own inherent caveats, as studies looking at reproducibility ${ }^{74}$ and modeling choices, ${ }^{13}$ reveal.

Noninvasive techniques such as ultrasound and magnetic resonance imaging are routinely used to provide us with a wide and versatile array of parameters. When referring to modeling, however, the relevance and direct comparison of simulation results with clinical observations should not be taken for granted in all cases. While much progress has been made with rigid wall models, the same is not true for anatomic cases at physiological conditions. CFD codes for carrying out high-resolution simulations in patientspecific geometries with compliant boundaries and at a resolution sufficiently fine to couple hemodynamics with biology or cellular biophysics either do not exist or are at early developmental stages. For example, FSI methods for simulating mechanical valves in anatomic configurations with compliant aorta and realistic boundary conditions from a left ventricle model are just now beginning to emerge. The same cautionary note holds for vascular structures and anomalies such as aneurysms, especially in the brain, where vessels are embedded close to surrounding soft and hard structures. The setting up of a computational model is inevitably connected with simplifications and departures from the full system under investigation, which sometimes implies that either the obtained results are qualitative and should be viewed as trends only, or that they reflect a selection of the pertinent physical and biological mechanisms and, again, should be viewed in this light.

We shall close this section by discussing briefly the issue of validation, an indispensable component of any computational simulation model. We observe that although in fluid mechanics (and consequently, in biofluids) validation and comparison with experiments are often straight-forward to instigate, such studies are not as commonplace as one would expect. A natural first step is of course the comparison with in vitro studies, frequently based on image-derived, anatomically accurate topologies, ${ }^{11}$ and ideally followed by comparisons with in vivo acquisitions. ${ }^{72}$ However, unless one is willing to accept commercial codes without much concern about the accuracy or physiologic relevance of the resulting flow fields, major emphasis should still be placed on developing and validating flow simulation tools that can predict the mechanical environment with the appropriate level of realism. In light of the recent trends towards multiscale/multiphysics models, validation becomes a significantly more involved issue; the complexity of the models implies that either individual components must be validated individually (useful in early stages but less so when the interactions between physical mechanisms and scales are studied) or that an appropriate "reduced system", where control can be exercised, is needed. Sometimes such a system may be an animal model, however in more cases than not, reduced systems are very difficult to devise and the only verification of the accuracy and predictive capability of the models is the comparison with the "full system", i.e., the patient and the specific pathology.

\section{FUTURE DIRECTIONS: TOWARDS INTEGRATIVE MULTI-SCALE AND MULTI-PHYSICS MODELS}

The application of sophisticated computational simulation models for the evaluation of the progress of disease, for the determination of long-term risk and for the planning of optimal treatment strategies is gaining ground in clinical practice: Personalized healthcare is a paradigm allowing for the enhancement of treatment specificity and efficacy. At the same time, continued progress in imaging helps reveal mechanisms and provides diagnostic possibilities that have not been available before.

The need for comprehensive, integrative models is gaining recognition. The terms "multiscale" and "multiphysics" are adequately descriptive of the direction this effort is taking. ${ }^{1,35}$ Many clinically relevant problems in cardiovascular biomedical engineering involve either spatially/temporally diverse scales or multiple mechanisms at intricate interplay with each other, or a combination of both. Examples like the following point up their prevalence in cardiovascular biomechanics: multi-bifurcation simulations, coupling of electrophysiology and perfusion with hemodynamics, the biochemistry of thrombosis, flow in microscopic regions of mechanical valves such as the valve hinges and the leakage jet during closure, wall remodeling, signaling pathways, and histology. They also underscore the importance that such complex mechanisms play in health and disease.

Although the majority of reported works in cardiovascular modeling involve the vasculature, there is evidence that more attention is being drawn to the heart 
itself. Multiphysics and multiscale modeling looking at the combination of coronary hemodynamics with the contractile motion of the myocardium, as triggered by the heart electrophysiology, is being reinforced as an important component of cardiovascular research and shows that the cardiac system is amenable to detailed modeling. ${ }^{54}$ Such multifaceted models, apart from the vast theoretical interest they present, can also serve as test beds for medication candidates and surgical treatments. Prominent examples of current research pursuits under this paradigm are the Cardiac Physiome ${ }^{4}$ and the Virtual Physiological Human (VPH) initiative ${ }^{70}$ that brings together several EU funded ICT-based projects for modeling and simulation of human physiology, such as the FP6 “@ neurIST" project ${ }^{30}$ (http://www. aneurist.org) and the recently started FP7 "euHeart" project $^{27}$ (http://www.euHeart.eu).

The inherent abundance of information provided by most integrative modeling approaches makes straining through simulation outcomes in search of clinically relevant indicators a formidable task in its own right. To put it differently, the translation of computational results to clinical conclusions involves a reductionist operation that at present is neither formalized nor of a globally acceptable nature. Scientific visualization of course plays a role in this effort, but more often than not, it is basic integral indicators that better convey the message and are needed.

The personalization of healthcare, a prevalent topic in the projections of experts on the developments expected in the near and mid-term future, constitutes an umbrella idea that covers many practical applications. An area where we expect to see imminent translation of computational techniques to clinical practice is the pre-interventional evaluation of the effectiveness of implants, involving comparing potential devices and selecting the best for the particular patient/lesion. With adequate progress, these integrated models can also represent a powerful tool for the computer-assisted surgery. Despite the promising outlook of patientspecific modeling in future healthcare applications, emphasis still needs to be placed on producing adequately validated predictive computational tools if the goal is to make a real impact on medical practice.

\section{CHALLENGES IN EMERGING HEALTHCARE APPLICATIONS}

The study of blood flow using computational techniques is developing at an accelerated pace. As we have discussed above, there are numerous studies entailing detailed comparisons and correlations of computed results with clinical observables; computations help elucidate underlying physiological or biophysical mechanisms, and, conversely, clinical observations guide and inspire the development of new models. Anatomically derived models of vasculature show great promise to deliver new levels of insight. However, progress is hindered in exactly those areas where the strength of such an approach would be expected. For example, we mentioned that there is a clear trend to make simulations a lot more multifactorial, multiscale, and multiphysics, and to include all the relevant mechanisms at play. In vascular biofluids, this inevitably means first incorporating the interaction of vascular wall dynamics, and vascular wall remodeling at a later stage. Although such approaches have already yielded valuable information on the basic processes at play when formulated around idealized vasculature models, developing them further to anatomically derived models leads to a direct and exigent need for additional subject-specific parameters. An often used example, pertinent to coupled blood-artery simulations, involves the need to provide information on vascular wall thickness, wall composition, and the perivascular environment. The latter, often overlooked, seems to be a critical factor in the distribution of loads on (at least certain segments of) vasculature. In any case, all three additional pieces of information, in more cases than not, are difficult to acquire. For certain large vessels, thickness is available non-invasively, but wall composition and, more importantly, mechanical properties of wall constituents are difficult to extract. In this combined imaging-modeling track of anatomically derived simulations, gaining access to such specificity is emerging as an important part of the big picture.

Although there has been very significant progress in the processing chains that are used in establishing anatomically accurate hemodynamics computations, the desired level of integration and automation is still missing. A key challenge for the near future is to establish a reliable, efficient, push-of-a-button tool for in vivo detailed blood flow quantification in clinical practice. The existence of such a suite constitutes an inexorable demand of the medical community, if computational models are to be adopted in a healthcare environment. These pursuits have to go hand-inhand with the development of advanced methods for data handling and display features of blood flow patterns that allow for analysis of large 3D data sets. Surprisingly, issues that where perceived as major obstacles in the not-so-distant past (like for example turnaround times for the number-crunching aspects of such models, or the availability of adequately versatile software for such simulations) seem to recede with the abundance and low-cost cluster parallel computing and affordable software suites for computational hemodynamics. 


\section{RECOMMENDATIONS}

The following steps are recommended to promote the translation to clinical applications in this rapidly evolving field:

- Strengthen bridges between computational models and physiology and vascular biology (cell signaling pathways in atherogenesis, rupture risk, remodeling)

- Encourage closer collaboration of basic life science and clinical medicine with imaging and biofluid engineers. Enhance communication and cross-fertilization by publishing biofluid mechanics books and/or review articles in clinical journals addressed to physicians. Host special sessions at clinical cardiology conferences (AHA/ACC), and invite clinicians to present keynote lectures at biofluid/engineering conferences.

- Incorporate basic biomedical engineering courses in medical curricula and offer training in physiological/biological modeling to biomedical engineers

- Maintain and deepen contacts with the medical device industry.

- Improve imaging (acquisition time, temporal and spatial resolution) and both anatomical and biofluid/biophysical feature extraction.

- Incorporate mechanical properties and surrounding structures/forces in CFD. Realistic parameter estimation (material, physiologic, biologic) requires an interdisciplinary approach. Experts from different disciplines should be brought together by organizing themed conferences.

- Validation-validation-validation... in vitro and in vivo.

\section{ACKNOWLEDGMENTS}

MS acknowledges support by the Netherlands Heart Foundation (Grants 2000.090, 2006B186) and by the European Community's Seventh Framework Programme (FP7/2007-2013) under Grant agreement No. 224495 (euHeart project). YV acknowledges support and inspiration from the EPSRC and the European Community's Sixth Framework Programme @neurIST.

\section{OPEN ACCESS}

This article is distributed under the terms of the Creative Commons Attribution Noncommercial License which permits any noncommercial use, distribution, and reproduction in any medium, provided the original author(s) and source are credited.

\section{REFERENCES}

${ }^{1}$ Antiga, L., M. Piccinelli, L. Botti, B. Ene-Iordache, A. Remuzzi, and D. Steinman. An image-based modeling framework for patient-specific computational hemodynamics. Med. Biol. Eng. Comput. 46:1097-1112, 2008.

${ }^{2}$ Artoli, A. M., A. G. Hoekstra, and P. M. A. Sloot. Mesoscopic simulations of systolic flow in the human abdominal aorta. J. Biomech. 39:873-884, 2006.

${ }^{3}$ Axner, L., A. G. Hoekstra, and P. M. A. Sloot. Simulating time harmonic flows with the lattice Boltzmann method. Phys. Rev. E Stat. Nonlin. Soft Matter Phys. 75:036709, 2007.

${ }^{4}$ Bassingthwaighte, J. B., P. J. Hunter, and D. Noble. The cardiac physiome- perspectives for the future. Exp. Physiol. 94:597-605, 2009.

${ }^{5}$ Bertram, C., and D. Gaver. Biofluid mechanics of the pulmonary system. Ann. Biomed. Eng. 33:1681-1688, 2005.

${ }^{6}$ Blackburn, H. M., S. J. Sherwin, and D. Barkley. Convective instability and transient growth in steady and pulsatile stenotic flows. J. Fluid Mech. 607:267-277, 2008.

${ }^{7}$ Bleasdale, R. A., K. H. Parker, and C. J. H. Jones. Chasing the wave. Unfashionable but important new concepts in arterial wave travel. Am. J. Physiol. Heart Circ. Physiol. 284:H1879-H1885, 2003.

${ }^{8}$ Borazjani, I. Numerical Simulations of Fluid-Structure Interaction Problems in Biological Flows. Ph.D. Thesis, University of Minnesota, Department of Mechanical Engineering, 2008.

${ }^{9}$ Borazjani, I., L. Ge, and F. Sotiropoulos. Curvilinear immersed boundary method for simulating fluid structure interaction with complex 3D rigid bodies. J. Comp. Phys. 227:7587-7620, 2008

${ }^{10}$ Borazjani, I., L. Ge, and F. Sotiropoulos. High-resolution fluid-structure interaction simulations of flow through a bileaflet mechanical heart valve in an anatomic aorta. Ann. Biomed. Eng., 2009 [Epub ahead of print]. doi:10.1007/ s10439-009-9807-x.

${ }^{11}$ Boutsianis, E., M. Guala, U. Olgac, S. Wildermuth, K. Hoyer, Y. Ventikos, and D. Poulikakos. CFD and PTV steady flow investigation in an anatomically accurate abdominal aortic aneurysm. J. Biomech. Eng. 131:011008 (15 pages), 2009. doi:10.1115/1.3002886.

${ }^{12}$ Caro, C. G., J. M. Fitzera, and R. C. Schroter. Arterial wall shear and distribution of early atheroma in man. Nature 223(5211):1159, 1969.

${ }^{13}$ Castro, M. A., C. M. Putman, and J. R. Cebral. Computational fluid dynamics modeling of intracranial aneurysms: effects of parent artery segmentation on intraaneurysmal hemodynamics. AJNR Am. J. Neuroradiol. 27:1703-1709, 2006.

${ }^{14}$ Chatziprodromou, I., D. Poulikakos, and Y. Ventikos. On the influence of variation in haemodynamic conditions on the generation and growth of cerebral aneurysms and atherogenesis: a computational model. J. Biomech. 40: 3626-3640, 2007.

${ }^{15}$ Chatziprodromou, I., A. Tricoli, D. Poulikakos, and Y. Ventikos. Haemodynamics and wall remodelling of a growing cerebral aneurysm: a computational model. J. Biomech. 40:412-426, 2007.

${ }^{16}$ Chen, C., H. Chen, D. Freed, R. Shock, I. Staroselsky, R. Zhang, A. Umit Coskun, P. H. Stone, and C. L. Feldman. Simulation of blood flow using extended Boltzmann kinetic approach. Phys. Stat. Mech. Appl. 362:174-181, 2006. 
${ }^{17}$ Chien, A., S. Tateshima, M. Castro, J. Sayre, J. Cebral, and F. Vinuela. Patient-specific flow analysis of brain aneurysms at a single location: comparison of hemodynamic characteristics in small aneurysms. Med. Biol. Eng. Comput. 46:1113-1120, 2008.

${ }^{18}$ Chien, S. Effects of disturbed flow on endothelial cells. Ann. Biomed. Eng. 36:554-562, 2008.

${ }^{19}$ Chopard, B., R. Ouared, and D. A. Rufenacht. A lattice Boltzmann simulation of clotting in stented aneursysms and comparison with velocity or shear rate reductions. Math. Comput. Simul. 72:108-112, 2006.

${ }^{20}$ Dasi, L. P., L. Ge, H. A. Simon, F. Sotiropoulos, and A. P. Yoganathan. Vorticity dynamics of a bileaflet mechanical heart valve in an axisymmetric aorta. Phys. Fluids 19:067105, 2007.

${ }^{21}$ David, T., and S. Moore. Modeling perfusion in the cerebral vasculature. Med. Eng. Phys. 30:1227-1245, 2008. doi: 10.1016/j.medengphy.2008.09.008.

${ }^{22}$ Davies, J. E., Z. I. Whinnett, D. P. Francis, C. H. Manisty, J. Aguado-Sierra, K. Willson, R. A. Foale, I. S. Malik, A. D. Hughes, K. H. Parker, and J. Mayet. Evidence of a dominant backward-propagating "Suction" Wave responsible for diastolic coronary filling in humans, attenuated in left ventricular hypertrophy. Circulation 113:1768-1778, 2006.

${ }^{23}$ Davies, P., J. Spaan, and R. Krams. Shear stress biology of the endothelium. Ann. Biomed. Eng. 33:1714-1718, 2005.

${ }^{24}$ Doorly, D., D. Taylor, A. Gambaruto, R. Schroter, and N. Tolley. Nasal architecture: form and flow. Philos. Transact. A Math. Phys. Eng. Sci. 366:3225-3246, 2008.

${ }^{25}$ Dumont, K., J. Vierendeels, R. Kaminsky, G. Van Nooten, P. Verdonck, and D. Bluestein. Comparison of the hemodynamic and thrombogenic performance of two bileaflet mechanical heart valves using a CFD/FSI model. J. Biomech. Eng. 129:558-565, 2007.

${ }^{26}$ Dwyer H. A., P. B. Matthews, A. Azadani, N. Jaussaud, L. Ge, T. S. Guy, and E. E. Tseng. Computational fluid dynamics simulation of transcatheter aortic valve degeneration. Interact. CardioVasc. Thorac. Surg. 9:301-308, 2009.

${ }^{27}$ Ecabert, O., and N. Smith. euHeart: integrated cardiac care using patient-specific cardiovascular modeling. SPIE Newsroom 1-3, 2008.

${ }^{28}$ Ethier, C. R., M. Johnson, and J. Ruberti. Ocular biomechanics and biotransport. Annu. Rev. Biomed. Eng. 6:249273, 2004.

${ }^{29}$ Figueroa, C. A., I. E. Vignon-Clementel, K. E. Jansen, T. J. R. Hughes, and C. A. Taylor. A coupled momentum method for modeling blood flow in three-dimensional deformable arteries. Comput. Meth. Appl. Mech. Eng. 195: 5685-5706, 2006.

${ }^{30}$ Frangi, A., D. R. Hose, and D. A. Ruefenacht. The @ neurist project: towards understanding cerebral aneurysms. SPIE Newsroom, 2007.

${ }^{31}$ Franklin, S. S. Beyond blood pressure: arterial stiffness as a new biomarker of cardiovascular disease. J. Am. Soc. Hypertens. 2:140-151, 2008.

${ }^{32}$ Ge, L., P. D. Dasi, F. Sotiropoulos, and A. P. Yoganathan. Characterization of hemodynamic forces induced by mechanical heart valves: Reynolds vs. Viscous stresses. Ann. Biomed. Eng. 36:276-297, 2008.

${ }^{33} \mathrm{Ge}$, L., and F. Sotiropoulos. A numerical method for solving the 3D unsteady incompressible Navier-Stokes equations in curvilinear domains with complex immersed boundaries. J. Comp. Phys. 225:1782-1809, 2007.

${ }^{34}$ Grigioni, M., C. Daniele, U. Morbiducci, C. Del Gaudio, G. D'Avenio, A. Balducci, and V. Barbaro. A mathematical description of blood spiral flow in vessels: application to a numerical study of flow in arterial bending. J. Biomech. 38:1375-1386, 2005.

${ }^{35}$ Grinberg, L., T. Anor, J. Madsen, A. Yakhot, and G. Karniadakis. Large-scale simulation of the human arterial tree. Clin. Exp. Pharmacol. Physiol. 36:194-205, 2009.

${ }^{36}$ Hadjiloizou, N., J. E. Davies, I. S. Malik, J. Aguado-Sierra, K. Willson, R. A. Foale, K. H. Parker, A. D. Hughes, D. P. Francis, and J. Mayet. Differences in cardiac microcirculatory wave patterns between the proximal left mainstem and proximal right coronary artery. Am. J. Physiol. Heart Circ. Physiol. 295:H1198-H1205, 2008.

${ }^{37}$ Harrison, S. E., J. Bernsdorf, D. R. Hose, and P. V. Lawford. A lattice Boltzmann framework for simulation of thrombogenesis. Progr. Comput. Fluid Dynam. Int. J. 8:121-128, 2008.

${ }^{38}$ Juvela, S. Treatment options of unruptured intracranial aneurysms. Stroke 35:372-374, 2004.

${ }^{39}$ Kakalis, N. M. P., A. P. Mitsos, J. V. Byrne, and Y. Ventikos. The haemodynamics of endovascular aneurysm treatment: a computational modelling approach for estimating the influence of multiple coil deployment. IEEE Trans. Med. Imaging 27:814-824, 2008.

${ }^{40}$ Katritsis, D. G., E. P. Efstathopoulos, J. Pantos, S. Korovesis, G. Kourlaba, S. Kazantzidis, V. Marmarelis, and E. Voridis. Anatomic characteristics of culprit sites in acute coronary syndromes. J. Interv. Cardiol. 21:140-150, 2008.

${ }^{41}$ Kharboutly, Z., M. Fenech, J. Treutenaere, I. Claude, and C. Legallais. Investigations into the relationship between hemodynamics and vascular alterations in an established arteriovenous fistula. Med. Eng. Phys. 29:999-1007, 2007.

${ }^{42}$ Kilner, P. J., G. Z. Yang, A. J. Wilkes, R. H. Mohiaddin, D. N. Firmin, and M. H. Yacoub. Asymmetric redirection of flow through the heart. Nature 404:759-761, 2000.

${ }^{43}$ Kurtcuoglu, V., M. Soellinger, P. Summers, K. Boomsma, D. Poulikakos, P. Boesiger, and Y. Ventikos. Computational investigation of subject-specific cerebrospinal fluid flow in the third ventricle and aqueduct of sylvius. J. Biomech. 40:1235-1245, 2007.

${ }^{44}$ LaDisa, Jr., J., L. Olson, H. Douglas, D. Warltier, J. Kersten, and P. Pagel. Alterations in regional vascular geometry produced by theoretical stent implantation influence distributions of wall shear stress: analysis of a curved coronary artery using 3D computational fluid dynamics modeling. Biomed. Eng. Online 5:40, 2006.

${ }^{45}$ Lawford, P. V., Y. Ventikos, A. W. Khir, M. Atherton, D. Evans, D. R. Hose, C. M. Care, P. N. Watton, I. Halliday, D. C. Walker, A. P. Hollis, and M. W. Collins. Modelling the interaction of haemodynamics and the artery wall: current status and future prospects. Biomed. Pharmacother. 62:530-535, 2008.

${ }^{46}$ Lee, S. W., L. Antiga, J. D. Spence, and D. A. Steinman. Geometry of the carotid bifurcation predicts its exposure to disturbed flow. Stroke 39:2341-2347, 2008.

${ }^{47}$ Lieber, B., M. Siebes, and T. Yamaguchi. Correlation of hemodynamic events with clinical and pathological observations. Ann. Biomed. Eng. 33:1695-1703, 2005.

${ }^{48}$ Mendoza, E., and G. W. Schmid-Schonbein. A model for mechanics of primary lymphatic valves. J. Biomech. Eng. 125:407-414, 2003.

${ }^{49}$ Millan, R. D., L. Dempere-Marco, J. M. Pozo, J. R. Cebral, and A. F. Frangi. Morphological characterization of intracranial aneurysms using 3-D moment invariants. IEEE Trans. Med. Imaging 26:1270-1282, 2007. 
${ }^{50}$ Moffatt, H. K., and A. Tsinober. Helicity in laminar and turbulent flow. Ann. Rev. Fluid Mech. 24:281-312, 1992.

${ }^{51}$ Morbiducci, U., R. Ponzini, M. Grigioni, and A. Redaelli. Helical flow as fluid dynamic signature for atherogenesis in aortocoronary bypass. A numeric study. J. Biomech. 40:519-534, 2007.

${ }^{52}$ Morbiducci, U., R. Ponzini, G. Rizzo, M. Cadioli, A. Esposito, F. De Cobelli, A. Del Maschio, F. Montevecchi, and $A$. Redaelli. In vivo quantification of helical blood flow in human aorta by time-resolved three-dimensional cine phase contrast magnetic resonance imaging. Ann. Biomed. Eng. 37:516-531, 2009.

${ }^{53}$ Narula, J., P. Garg, S. Achenbach, S. Motoyama, R. Virmani, and H. W. Strauss. Arithmetic of vulnerable plaques for noninvasive imaging. Nat. Clin. Pract. Cardiovasc. Med. 5:S2-S10, 2008.

${ }^{54}$ Niederer, S. A., and N. P. Smith. An improved numerical method for strong coupling of excitation and contraction models in the heart. Prog. Biophys. Mol. Biol. 96:90-111, 2008.

${ }^{55}$ Ohayon, J., O. Dubreuil, P. Tracqui, S. Le Floc'h, G. Rioufol, L. Chalabreysse, F. Thivolet, R. Pettigrew, and G. Finet. Influence of residual stress/strain on the biomechanical stability of vulnerable coronary plaques: potential impact for evaluating the risk of plaque rupture. Am. J. Physiol. Heart Circ. Physiol. 293:H1987-H1996, 2007.

${ }^{56}$ Ohayon, J., G. Finet, A. M. Gharib, D. A. Herzka, P. Tracqui, J. Heroux, G. Rioufol, M. S. Kotys, A. Elagha, and R. I. Pettigrew. Necrotic core thickness and positive arterial remodeling index: emergent biomechanical factors for evaluating the risk of plaque rupture. Am. J. Physiol. Heart Circ. Physiol. 295:H717-H727, 2008.

${ }^{57}$ Parker, K. H. An introduction to wave intensity analysis. Med. Biol. Eng. Comput. 47:175-188, 2009.

${ }^{58}$ Parthasarathi, A. A., K. Grosh, and A. L. Nuttall. Threedimensional numerical modeling for global cochlear dynamics. J. Acoust. Soc. Am. 107:474-485, 2000.

${ }^{59}$ Prasad, A., and S. Tsimikas. Candidate biomarkers for the detection of coronary plaque destabilization and rupture. Curr. Atheroscler. Rep. 10:309-317, 2008.

${ }^{60}$ Radaelli, A. G., L. Augsburger, J. R. Cebral, M. Ohta, D. A. Rufenacht, R. Balossino, G. Benndorf, D. R. Hose, A. Marzo, R. Metcalfe, P. Mortier, F. Mut, P. Reymond, L. Socci, B. Verhegghe, and A. F. Frangi. Reproducibility of haemodynamical simulations in a subject-specific stented aneurysm model - a report on the Virtual Intracranial Stenting Challenge 2007. J. Biomech. 41:2069-2081, 2008.

${ }^{61}$ Ricotta, J., J. Pagan, M. Xenos, Y. Alemu, S. Einav, and D. Bluestein. Cardiovascular disease management: the need for better diagnostics. Med. Biol. Eng. Comput. 46:10591068, 2008.

${ }^{62}$ Rybicki, F. J., S. Melchionna, D. Mitsouras, A. U. Coskun, A. G. Whitmore, M. Steigner, L. Nallamshetty, F. G. Welt, M. Bernaschi, M. Borkin, J. Sircar, E. Kaxiras, S. Succi, P. H. Stone, and C. L. Feldman. Prediction of coronary artery plaque progression and potential rupture from 320-detector row prospectively ECG-gated single heart beat CT angiography: lattice Boltzmann evaluation of endothelial shear stress. Int. J. Cardiovasc. Imaging 25(suppl 2):289-299, 2009.

${ }^{63}$ Shipkowitz, T., V. Rodgers, L. Frazin, and K. Chandran. Numerical study on the effect of secondary flow in the human aorta on local shear stresses in abdominal aortic branches. J. Biomech. 33:717-728, 2000.
${ }^{64}$ Siggers, J. H., S. Waters, J. Wattis, and L. Cummings. Flow dynamics in a stented ureter. Math. Med. Biol. 26:124, 2009.

${ }^{65}$ Sotiropoulos, F., and I. Borazjani. A review of the state-ofthe-art numerical methods for simulating flow through mechanical heart valves. Med. Biol. Eng. Comput. 47:245256, 2009.

${ }^{66}$ Spaan, J., C. Kolyva, J. van den Wijngaard, R. ter Wee, P. van Horssen, J. Piek, and M. Siebes. Coronary structure and perfusion in health and disease. Philos. Transact. A Math. Phys. Eng. Sci. 366:3137-3153, 2008.

${ }^{67}$ Spaan, J. A. E., J. J. Piek, J. I. E. Hoffman, and M. Siebes. Physiological basis of clinically used coronary hemodynamic indices. Circulation 113:446-455, 2006.

${ }^{68}$ Steinman, D., and C. Taylor. Flow imaging and computing: large artery hemodynamics. Ann. Biomed. Eng. 33: 1704-1709, 2005.

${ }^{69}$ Steinman, D. A., J. S. Milner, C. J. Norley, S. P. Lownie, and D. W. Holdsworth. Image-based computational simulation of flow dynamics in a giant intracranial aneurysm. AJNR Am. J. Neuroradiol. 24:559-566, 2003.

${ }^{70}$ STEP Consortium. Seeding the EuroPhysiome: a roadmap to the virtual physiological human, [Online] 5 July 2007: http://www.europhysiome.org/roadmap.

${ }^{71}$ Sugawara, M., K. Niki, N. Ohte, T. Okada, and A. Harada. Clinical usefulness of wave intensity analysis. Med. Biol. Eng. Comput. 47:197-206, 2009.

${ }^{72}$ Sundareswaran, K. S., D. H. Frakes, M. A. Fogel, D. D. Soerensen, J. N. Oshinski, and A. P. Yoganathan. Optimum fuzzy filters for phase-contrast magnetic resonance imaging segmentation. J. Magn. Reson. Imaging 29:155$165,2009$.

${ }^{73}$ Suo, J., J. Oshinski, and D. Giddens. Blood flow patterns in the proximal human coronary arteries: relationship to atherosclerotic plaque occurrence. Mol. Cell. Biomech. 5:918, 2008.

${ }^{74}$ Thomas, J. B., J. S. Milner, B. K. Rutt, and D. A. Steinman. Reproducibility of image-based computational fluid dynamics models of the human carotid bifurcation. Ann. Biomed. Eng. 31:132-141, 2003.

${ }^{75}$ Van Tricht, I., D. De Wachter, J. Tordoir, and P. Verdonck. Comparison of the hemodynamics in $6 \mathrm{~mm}$ and 4-7 mm hemodialysis grafts by means of CFD. J. Biomech. 39:226-236, 2006.

${ }^{76}$ Watton, P. N., N. A. Hill, and M. Heil. A mathematical model for the growth of the abdominal aortic aneurysm. Biomech. Model Mechanobiol. 3:98-113, 2004.

${ }^{77}$ Watton, P. N., Y. Ventikos, and G. A. Holzapfel. Modelling growth and stabilisation of cerebral aneurysms. Math. Med. Biol. 26(2):133-164, 2009.

${ }^{78}$ Weir, B. Unruptured intracranial aneurysm: a review. J. Neurosurg. 96:3-42, 2002.

${ }^{79}$ Wilson, W., C. C. van Donkelaar, B. van Rietbergen, and R. Huiskes. A fibril-reinforced poroviscoelastic swelling model for articular cartilage. J. Biomech. 38:1195-1204, 2005.

${ }^{80}$ Xiong, G., J. Zhan, K. Zuo, J. Li, L. Rong, and G. Xu. Numerical flow simulation in the post-endoscopic sinus surgery nasal cavity. Med. Biol. Eng. Comput. 46:11611167, 2008.

${ }^{81}$ Yamashita, S., H. Isoda, M. Hirano, H. Takeda, S. Inagawa, Y. Takehara, M. T. Alley, M. Markl, N. J. Pelc, and H. Sakahara. Visualization of hemodynamics in intracranial arteries using time-resolved three-dimensional phasecontrast MRI. J. Magn. Reson. Imaging 25:473-478, 2007. 